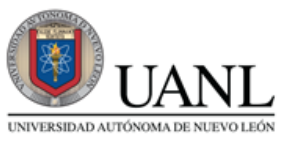

FACPYA
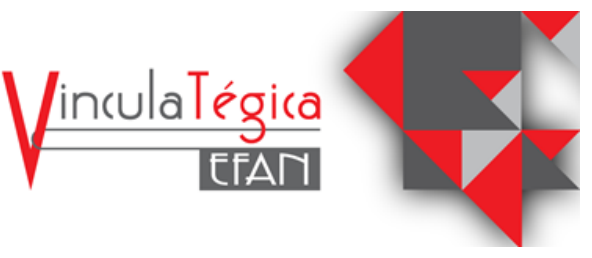

\title{
Formación e identidad de emprendedores en tiempos de crisis: Aportes de un marco conceptual y metodológico
}

\author{
Gabriela Croda Borges ${ }^{1}$ y Ana Laura Domínguez Paredes ${ }^{2}$ \\ 1Universidad Popular Autónoma del Estado de Puebla, Facultad de Posgrados, Puebla, Puebla, México, \\ gabriela.croda@upaep.mx, 17 Sur 9013, Barrio de Santiago, C.P. 72410, Puebla, Pue., México. Tel.: +52 \\ (222)2299400 Ext. 7671 \\ 2Universidad Popular Autónoma del Estado de Puebla, México, Facultad Económico-Administrativo, \\ analaura.dominguez01@upaep.mx, 11 Poniente 2309, Barrio de Santiago, C.P. 72410, Puebla, Pue., México. \\ Tel.: +52 (222)2299400 Ext.7884
}

Información del artículo revisado por pares

Fecha de aceptación: junio-2021

Fecha de publicación en línea: diciembre-2021

DOI: https://doi.org/10.29105/vtga7.2-51

\section{Resumen}

La actual crisis sanitaria de Covid-19 en México ha tenido un impacto importante en las micro, pequeñas y medianas empresas (MiPymes) ocasionando que varias de ellas hayan tenido que cerrar operaciones y en casos extremos despedir a su personal.

Sumado a las actuales políticas públicas sobre la agenda de desarrollo económico en nuestro país, sobre el apoyo para las MiPymes y de los emprendedores aún no es muy claro, dejando a varios de estos emprendedores en solitud con muy nulo apoyo. Si bien, surgieron varios seminarios de manera gratuita, apoyos económicos emergentes algunos de ellos no lograron sobrevivir como se mencionó anteriormente. Por lo mismo, la intención de la siguiente investigación es aportar un marco conceptual y metodológico que ayude a identificar la situación actual de las empresas y/o emprendimientos desde la formación para fortalecer aquellas áreas de oportunidad y generar una visión a futuro que ayude a ser mucho más resilientes y mejor preparados frente a otras crisis.

Palabras clave: Crisis sanitaria, Emprendimiento, Formación de Emprendedores, MiPymes.

\begin{abstract}
The current Covid-19 health crisis in Mexico has had a significant impact on micro, small and medium-sized companies (MiPymes) causing several of them to close operations and in extreme cases lay off their staff.

In addition to current public policies on the economic development agenda in our country, the support for MSMEs and entrepreneurs is still not very clear, leaving several of these entrepreneurs alone with none support. Although several seminars and loans emerged, enterprises did not survive.

Therefore, the intention of the following research is to provide a conceptual methodological framework that helps them identify the current situation of their companies and / or ventures for training to strengthen those areas of opportunity and generate a vision for the future that helps them to be much more resilient and be better prepared to face other crisis in the near future.
\end{abstract}

Keywords: Health Crisis, Entrepreneurship, SMBs, Entrepreneurship training, MSMEs .

JEL: D99 - Otros 


\section{INTRODUCCIÓN}

La pandemia global de Covid-19, ha tenido un impacto social, económico y medioambiental en nuestro país importante. Según, Gámez, M. (2019) en la actual agenda presidencial, se busca fortalecer el desarrollo económico para el primer año pasando de $1.5 \%$ al $2.5 \%$ para que al finalizar el sexenio se logre un crecimiento del $4 \%$, sin embargo, dentro de las actividades para esta agenda el impulso para la pequeña y mediana empresa aún es un tema pendiente, sumado a la desaparición del Instituto Nacional del Emprendedor (INADEM), México se encuentra en mayor desventaja que otras economías que centran sus esfuerzos hacia la innovación y el fortalecimiento empresarial para los pequeños y medianos empresarios colocando a nuestro país en una mayor desventaja.

Según, Navarrete, F. (2020) y con base en cifras del INEGI, las pequeñas y medianas empresas del país representan el 99\% del total operando en México, las cuales generan $72 \%$ del empleo y el 52\% del Producto Interno Bruto (PIB) y son las que emplean entre el $70 \%$ y $80 \%$ del nuevo empleo. Su modo de operación diario, se basa en la supervivencia, sin mucha oportunidad para contar con una planeación de largo plazo, la profesionalización de su empresa e inversión en capital y tecnología. Sumado a lo anterior y con la situación actual de salud pública, varias de ellas tuvieron que cerrar operaciones y realizar despidos de su personal dejando a muchas personas sin empleo. Las industrias más afectadas han sido las de entretenimiento, alojamiento y turística.

Ante esta situación que implica un gran reto en nuestro país, este trabajo de investigación surge a partir de la necesidad de revisar la literatura sobre ¿Cuáles han sido los mayores retos que los emprendedores están enfrentando ante esta crisis sanitaria? ¿De qué manera podemos apoyar a los emprendedores desde la perspectiva de la formación en emprendimiento?

\section{MARCO TEÓRICO}

\subsection{Impacto Covid-19 en las Empresas}

De acuerdo al Banco Mundial, Aguilar, (2020) señala que, los países con ingresos bajos y medios como México su crecimiento del PIB será negativo en los siguientes ámbitos muy estratégicos para nuestra economía: El turismo, comercio, la productividad y a las Mipymes que contratan a muchas personas para la fuerza laboral y que debido a la crisis económica varias de ellas se verán gravemente afectadas por el despido de su personal, entre otros factores. En México, existen más de 4.5 millones de Mipymes, este conjunto de empresas emplea el $78 \%$ de los empleos formales en el país. De los 4.5 millones de unidades económicas que pertenecen a las empresas micro, éstas ocupan entre 1 y 10 empleos; las pequeñas emplean entre 10 y 50 trabajadores, y las medianas contratan entre 50 y 500 trabajadores.

Por otra parte, Esquivel (2020), enfatizó que el impacto del Covid en la economía se dio en tres etapas: la primera a finales de Marzo del 2020 en la que hubo cancelación de vuelos a nivel internacional, impactando al sector turístico. La segunda etapa, cuando los establecimientos de actividades no esenciales cerraron y la que más afectó a la industria y al comercio. Por último, la tercera fase, se refiere a la reapertura de los establecimientos que es en la etapa en la que se encuentra nuestro país.

El Grupo SMB (firma de consultoría en adopción de tecnología y tendencias en las pequeñas y medianas empresas (SMB) asentada en Boston, E.U.A.) realizó a los empresarios un estudio en el que los microempresarios estimaron tener un $60 \%$ de reducción de sus ingresos, en un $30 \%$ en los próximos seis meses; el $3 \%$ cerró definitivamente y un $43 \%$ reportó que han cerrado temporalmente con la expectativa de reabrir. El 34\% de las Pymes ya iniciaron la reducción de sueldos y de horas de trabajo; el $24 \%$ planea despedir o reducir las horas de los empleados asalariados, y el $22 \%$ tiene la intención de hacerlo por medio de asalariados. Para apoyar a las Pymes y lograr preservar los empleos, el gobierno de Estados Unidos destinó alrededor de \$350,000 millones de 
dólares y en México, el sector público destinó $\$ 25,000$ millones de pesos para créditos a un millón de Mipymes y un millón para aquellas que no hayan despedido a su personal. Asimismo, también surgieron algunas iniciativas de plataformas que daban orientación a los pequeños empresarios, como: Asesorías en línea, vídeo cursos y talleres o webinars para ayudar a los emprendedores y empresarios a reactivarse. Sin embargo, muchas de estas iniciativas no lograron evitar la pérdida significativa del desempleo, cierre de sus empresas y agravar el tema de pobreza en el país. (Maraboto, 2020)

\subsection{Emprendimiento y Emprendedor tiempo de Crisis}

Rodríguez (2020), menciona en su artículo de "Cómo emprender en tiempo de crisis", la importancia de las crisis para hacer los ajustes a la economía y abrir nuevas oportunidades. Son tiempos para cuidar de los empleados internos y externos además de reflexión para detonar nuevos procesos, ideas y negocios incluso. Enfatiza que la diferencia está en adaptarse a las circunstancias, el diseño e implementación de estrategias adecuadas para no morir en el intento.

Asimismo, Martínez, N., y Rubio, B. (2020) afirman la importancia que tiene el emprendimiento en tiempo de crisis porque ayuda a reacomodar lo viejo, lo que se encontraba en su lugar, en tiempo de crisis, se mueve ya sea para crear nuevas industrias, productos y/o servicios y mercados. Del mismo modo, se abren oportunidades para el empleo y la creación de nuevas empresas.

El rol del emprendedor en tiempo de crisis es de suma importancia, ya que, por medio de su creatividad funge como ese catalizador de cambio en los mercados por lo mismo es importante conocer a qué tipo de actividad económica se dedica. Las investigadoras, Martínez, N., y Rubio, B. (2020) citan que Leibenstein (1968) distingue dos tipos de actividad empresarial que desempeñan los empresarios: En un extremo la actividad empresarial rutinaria basada en la apegada a procesos y del otro extremo se encuentra la actividad empresarial Schumpeteriana o de nueva creación. Muñoz, Palacios (2017) menciona que existen determinadas características personales que los emprendedores poseen: autoconfianza para convertir su idea en un proyecto real; resiliencia y perseverancia; talento para identificar oportunidades en su entorno y capitalizarlas y conocimientos de negocio para comercializar su producto en el mercado.

Algunos de los retos a los que se han enfrentado los empresarios son en las áreas de acceso de capital de inversión debido en parte al detenimiento parcial de las actividades económicas, lo cual detonó incertidumbre y elevó el riesgo de sus inversiones. Ordaz, Díaz (2019) por lo mismo los empresarios han solicitado al sector público una mayor inversión y confianza en invertir. Durante octubre de 2019, la confianza empresarial disminuyó 0.2 puntos en el sector de la manufactura, en el de comercio de 0.8 puntos y de menos 0.7 en el de la construcción, de acuerdo con cifras del Instituto Nacional de Estadística y Geografía.

Otro de los aspectos en el que se vieron afectados los emprendedores y empresarios como señala De la Vega y Lorenzo (2020) fue la baja de la demanda y alargamiento de sus ciclos de venta, incertidumbre, el pago de impuestos, retrasos en suministros, temas relacionados con el crédito y los cobros de sus cuentas por cobrar y afectación en su capacidad de producción debido a las restricciones sanitarias. La digitalización permitió en algunas industrias no perder el contacto con sus clientes y continuar con las actividades laborales desde casa.

\subsection{Necesidades de formación en el contexto actual}

Dado lo anterior y asumiendo en un escenario futuro la posibilidad de nuevas crisis o emergencias sanitarias y/o medioambientales, se considera importante en la formación del emprendedor, así como, desarrollar su pensamiento crítico para la toma de decisiones en situación de cambios y formarlo en su 
autoconfianza para potenciar su espíritu creador.

Con base en resultados de investigaciones, se puede afirmar que en el actual contexto de crisis existen necesidades y demandas sociales, económicas y culturales que exigen un perfil profesional renovado, que en el que se incluyan competencias tales como la capacidad de innovar, la mediación, el trabajo colaborativo, la creatividad, las habilidades comunicativas, la disposición al cambio entre otros. Lo anterior, desde el reconocimiento de la necesidad de formar personas de manera integral, que atienden las necesidades del entorno e intervengan en el contexto con sentido de responsabilidad y compromiso social. Para ello, se considera que las metodologías educativas disruptivas, innovadoras, activas y creativas, pueden representan un dispositivo pedagógico que contribuye a una formación que supera los saberes conceptuales o declarativos y favorece la formación de emprendedores con sentido social a través experiencias educativas que resulten significativas mediante la vivencia que promueva el desarrollo de competencias emprendedoras para afrontar un futuro de incertidumbre.

Los ambientes educativos son fundamentales para el desarrollo del emprendedor, por lo que es indispensable incluir las dimensiones personal y social en la formación de emprendedores, así como situar en el contexto y momento histórico las experiencias formativas, considerando la relevancia del entorno en el desarrollo de las competencias del emprendedor, particularmente en el contexto de crisis. En este sentido, es relevante considerar que la formación de emprendedores implica la articulación de la propuesta formativa, la cultura emprendedora y las competencias para el emprendimiento con la intencionalidad de formar emprendedores con actitudes innovadoras y creativas transferibles a otros contextos de forma idónea y sentido ético.

El contexto actual en el que los efectos de la crisis sanitaria, requieren una reactivación económica, plantea desafíos importantes para la formación de emprendedores con una amplia comprensión de las necesidades socio formativas de los emprendedores, destacando la dimensión personal y social, en donde los aspectos socioemocionales, cobran relevancia, así como la necesidad de situar los procesos de formación en contextos reales, abordar una visión integral y sistémica, así como atender el componente ético tanto en sentido de los bienes que derivan del emprendimiento como del desarrollo profesional del emprendedor.

La formación de emprendedores, en en el contexto de la crisis sanitaria, requiere ampliar la visión centrada en la creación y gestión de empresas y orientarse a promover la motivación de los futuros emprendedores como agentes de cambio de la realidad social a través de los emprendimientos. Para ello, es imperativo repensar la formación de emprendedores para superar la visión economicista del emprendimiento y plantear una perspectiva sistémica y contextualizada de formación para el emprendimiento con enfoque social

Para fortalecer la formación integral de los emprendedores, es fundamental que tanto en la universidad, como en los programas de emprendimiento y en las incubadoras de empresas, agentes formadores $\mathrm{y}$ transformadores, se promueva la vivencia de procesos de formación que reconozcan las expectativas, creencias, intenciones e intereses personales, así como, las condiciones del entorno que actúan facilitando $u$ obstaculizando los emprendimientos.

Desde dichos referentes se considera que las experiencias educativas para el emprendimiento deben ser significativas e interpelar las dimensiones de la persona en sentido integral. En ese sentido, resulta imprescindible propiciar de forma intencionada procesos para el encuentro, la construcción y vivencia de situaciones en contextos auténticos que movilicen los saberes de la persona para el desarrollo de las competencias emprendedoras que sean transferibles y situadas.

\subsection{Identidad emprendedora}

En el contexto complejo e incierto generado por la crisis sanitaria, se hace 
necesario fortalecer los procesos de formación que contribuyan al desarrollo de identidades que se enriquezcan con el aprendizaje, de ahí que con base en el desarrollo de las habilidades y la construcción de conocimientos, pueden participar con mayor dominio en los contextos en donde se sitúan los emprendimientos, de forma que se vayan transformando las identidades, es decir, "los cambios que resultan de su acrecentada destreza lo transforman como individuo en un sentido amplio." (Zurlinden, 2010, p. 7).

La construcción de la identidad constituye un proceso múltiple, variable y dinámico en el que se desarrollan las dimensiones de la persona de forma interrelacionada, en la que se vinculan las acciones con las relaciones en contextos sociales e históricos determinados, que se construye como un concepto diferencial, como un proceso, que implica movilidad, transformación y cambio (Navarrete, 2008)

La construcción de la identidad incluye el reconocimiento de la forma en que cada persona se constituye como persona, en su vivir, actual y pensar; implica las representaciones sociales que la persona hace de sí misma y del entorno con base en los roles y posiciones que ocupa (Navarrete, 2007).

El concepto de identidad supone al mismo tiempo la idea de "singularidad", de diferenciación del resto, y el de "pertenencia" a determinados grupos. La construcción de la identidad obedece a un proceso dinámico y evolutivo, en contextos de interacción social (Bernal, 2014), como lo es el contexto en el que se desarrolla el emprendedor.

En este sentido, se considera que las personas construyen su identidad cuando asumen distintas posiciones o roles, (Navarrete, 20015), ya que una persona a lo largo de la vida desempeña diversos roles, con lo que la identidad se reconstruye constantemente renovando los roles y sus significaciones.

La situación actual del Covid-19 impulsa a las personas a realizar sobre sí mismo, para el desarrollo de nuevas competencias que le permitan responder a las necesidades sociales y demandas del mercado, en las actuales condiciones, a fin de construir un polo identitario emprendedor que atienda a los desafíos del actual contexto, así como a las necesidades personales, profesionales, sociales, económicas y culturales.

La identidad emprendedora se asume como "el proceso multidimensional de construcción personal e identificación axiológica, dinámico e inacabado que se desarrolla al vivir situaciones con determinadas características y condiciones en contextos de interacción social, para integrar las expectativas personales y profesionales con las experiencias vividas para favorecer la comprensión del sentido y alcance de la competencia emprendedora" (Croda y Sánchez, 2019, p.7)

\section{5. Formación del emprendedor en tiempos de crisis}

La situación actual, debida a la contingencia sanitaria de Covid-19, impulsa a las personas a salir de su zona de confort, asumir riesgos, adaptarse al cambio, reconocer $\mathrm{y}$ atender las oportunidades en el proceso continuo de su desarrollo profesional, en la búsqueda de atender las competencias de tipo socioemocional para responder a las nuevas necesidades y demandas, enmarcadas en un contexto de crisis.

\section{MÉTODO}

El método es cualitativo de tipo descriptivo y con base en criterios de revisión sistemática mediante el uso de matriz heurística a fin de determinar las categorías para identificar las necesidades de formación y sus procesos identitarios en tiempos de crisis durante la pandemia del Covid-19 y los retos a los cuales se enfrentan. Los resultados que se presentan derivan de la revisión de literatura y la selección de las investigaciones relativas a la formación de los emprendedores, el emprendimiento, la construcción de la identidad y la categoría de los desafíos a los que los emprendedores y/o empresarios se enfrentaron durante la pandemia de Covid-19. 


\section{RESULTADOS}

Con base en la revisión de la literatura se integró la matriz heurística de categorías para la construcción del instrumento de recopilación de información en la siguiente fase del estudio.

Se identificaron tres categorías para el estudio: retos que enfrentaron las Pymes ante el Covid19, identidad emprendedora y necesidades formativas para el emprendimiento.

Las categorías se definieron conceptualmente de la siguiente manera:

Retos que enfrentaron las Pymes ante el Covid-19, entendida como: Se entiende con base en los autores Rodríguez (2020), Martínez, N., y Rubio, B, 2020, Palacios (2017), Ordaz, Díaz (2019), De la Vega y Lorenzo (2020), Esquivel (2020), Maraboto, 2020) por retos aquellos aspectos por los cuales el emprendedor y/o empresario tuvieron que hacer frente ante la situación del antes, durante post pandemia del Covid 19.

La categoría identidad emprendedora (Croda, Sánchez, 2019, p. 5,) "se refiere al proceso multidimensional de construcción personal e identificación axiológica, dinámico e inacabado que se desarrolla al vivir situaciones con determinadas características y condiciones en contextos de interacción social, para integrar las expectativas personales y profesionales con las experiencias vividas para favorecer la comprensión del sentido y alcance de la competencia emprendedora", con fundamento en Glade (1969), Roberts y Wood (2005), Cantón, Luit y Ramirez (2008), Vázquez y Dávila (2008), Bernal (2014) y Navarrete (2015), Martínez, N., y Rubio, B. (2020)

La categoría necesidades formativas para el emprendimiento, referida a las necesidades de formación para el emprendimiento que se reconocen a partir de las competencias que se requieren desarrollo en la persona mediante procesos que favorecen el encuentro, la construcción y vivencia de situaciones que movilizan recursos para el desarrollo de competencias emprendedoras para enfrentar las condiciones del contexto de la pandemia por Covid-19, teniendo como referentes teóricos a: Contreras y Pérez (2013), de Camilloni, (s/f), Ramirez (2015), Osorio y Pereira (2011)

Por cada categoría definida se identificaron desde la literatura, los descriptores, que dieron la pauta para la formulación de las preguntas que permitirán identificar las necesidades formativas de emprendedores durante la pandemia de Covid 19 y la manera en que han incidido en su identidad emprendedora.

A continuación se analizan los descriptores obtenidos como resultado de la revisión documental por cada una de las categorías.

Dentro de la categoría de Retos que enfrentaron las Pymes ante el Covid-19 se encontraron los siguientes: Acceso a capital, el cual se refiere si el empresario recibió algún tipo de apoyo por parte del gobierno y se tomó en cuenta si, este apoyo está en espera, denegado, no lo ha solicitado o si está recibiendo ayuda.

Dentro de la categoría de la demanda se identificó si las ventas aumentaron o disminuyeron durante esta crisis, si dejó de vender o si se ha mantenido estable.

Dentro de la categoría de operaciones se encontró si el empresario tuvo contratiempos para la obtención de la materia prima, en caso de ser afirmativa la respuesta, conocer si tuvo que recurrir a sustitutos, dónde los obtuvo y si se elevó el precio de los mismos.

En cuanto a la categoría de digitalización, las variables son si la empresa ya se cuenta digitalizada, en caso de ser que sí, ¿Cuál? y si es negativa la respuesta, ¿Por qué no accedió a la digitalización?

Finalmente, en cuanto a la categoría de personal, se encuentran aquellas variables relacionadas con la rescisión de contratos, reducción de horas laborales, disminución de sueldos, contratación de personal, y/o mantención de nómina.

En la categoría de identidad emprendedora se encontraron las variables de si el emprendedor se reconoce a sí mismo, si se diferencia de otros tipos de emprendedores, cómo maneja el stress y las actitudes en tiempo de crisis, lo cual refleja la importancia de la 
dimensión personal en la conformación de la identidad emprendedora.

En cuanto a la categoría de necesidades formativas para el emprendimiento, se encontró: si obtuvo formación durante y post pandemia en alguno de estos temas: financiero, impuestos, recursos humanos, manejo de stress, ¿cuál fue la experiencia educativa más significativa o enriquecedora durante tu formación emprendedora? y ¿por qué?, ¿cuál cree que fue la intención de realizar estas actividades como parte de su formación emprendedora? ¿qué fue lo más importante para usted? y finalmente, ¿cuál fue el propósito que tuvo para dicha actividad?, en este sentido, los tipos de necesidades de formación se ampliaron y diversificaron a raíz de la contingencia sanitaria.

Durante la siguiente fase de la investigación, se realizará el proceso de validación y prueba piloto del instrumento, para posteriormente proceder a la realización de las entrevistas y el análisis de la información.

Las preguntas de la guía de entrevista son:

1. ¿Recibió algún tipo de apoyo por parte del gobierno?

2. ¿Sus ventas han aumentado?

3. ¿Sus ventas han disminuido?

4. ¿Dejó de vender?

5. ¿Se ha mantenido constante?

6. ¿Tuvo retrasos al adquirir sus suministros?

7. En caso de que la respuesta sea afirmativa, ¿Tuvo que buscar sustitutos? ¿Dónde los obtuvo?

8. ¿Se elevó el precio?

9. ¿Su empresa ya contaba con algún servicio de digitalización?

10. Si, No.

11. En caso afirmativo, ¿Cuál?

12. En caso negativo, ¿Platícanos qué fue lo que hizo que no accediera a la parte de la digitalización?
13. ¿Se vio en la necesidad de rescindir contratos laborales a personas dentro de su empresa?

14. ¿Mantuvo la nómina?

15. ¿Se disminuyeron los salarios?

16. ¿Se contrató a más personal?

17. ¿Reconoces tu perfil emprendedor?

18. ¿Cuál es?

19. ¿Qué características tiene?

20. ¿Cómo se diferencia de otros tipos de emprendedores?

21. ¿Cómo maneja el stress?

22. ¿Qué actitud toma ante las crisis?

23. ¿Tuvo acceso a algún tipo de formación durante y post pandemia?

24. ¿Cuál fue la experiencia educativa más significativa o enriquecedora durante tu formación emprendedora? y ¿Por qué?

25. ¿Cuál cree que fue la intención de realizar estas actividades como parte de su formación emprendedora?

26. ¿Qué fue lo más importante para usted?

27. ¿Cuál fue el propósito que tuvo para dicha actividad?

\section{CONCLUSIONES}

Para seguir fortaleciendo las competencias del emprendedor y/o empresario es necesario abonar a su formación en emprendimiento para incrementar su identidad emprendedora en apoyo a su crecimiento personal, profesional y social. En la medida en la que un emprendedor y/o empresario abone a su formación su crecimiento será reflejado en los resultados de su empresa.

Los aspectos socioemocionales de identidad recobran hoy más que nunca una mayor importancia en la formación de los emprendedores, por lo que indagar sobre sus necesidades de formación en tiempos de crisis, inciertos y complejos, resulta imperativo.

En prospectiva de la investigación, se realizará el estudio empírico, partiendo de la validación del instrumento mediante el juicio 
de expertos en el que se indague sobre las categorías identificadas en el análisis de la literatura: retos que enfrentaron las Pymes ante el Covid-19, identidad emprendedora y necesidades formativas para el emprendimiento.

Tabla 1. Matriz heurística de categorías de estudio

\begin{tabular}{|c|c|c|c|}
\hline Categoría & Descripción Conceptual & Descriptores & Preguntas \\
\hline \multirow[t]{5}{*}{$\begin{array}{l}\text { Retos que enfrentaron las } \\
\text { Pymes ante el Covid- } 19\end{array}$} & \multirow{5}{*}{$\begin{array}{l}\text { Se entiende por retos } \\
\text { aquellos aspectos por los } \\
\text { cuales el emprendedor y/o } \\
\text { empresario tuvieron que } \\
\text { hacer frente ante la situación } \\
\text { del antes, durante post } \\
\text { pandemia del Covid } 19 \text {. } \\
\text { Rodríguez (2020), Martínez, } \\
\text { N., y Rubio, B, 2020), } \\
\text { Palacios (2017), Ordaz, } \\
\text { Díaz (2019), De la Vega y } \\
\text { Lorenzo (2020), Esquivel } \\
\text { (2020), Maraboto, 2020) }\end{array}$} & Acceso a Capital & $\begin{array}{l}\text { ¿Recibió algún tipo de apoyo por } \\
\text { parte del gobierno? } \\
\text {-En espera } \\
\text {-Denegada } \\
\text {-No ha solicitado } \\
\text {-Está recibiendo ayuda }\end{array}$ \\
\hline & & Demanda & $\begin{array}{l}\text { ¿Sus ventas han aumentado? } \\
\text { ¿Sus ventas han disminuido? } \\
\text { ¿Dejó de vender? } \\
\text { ¿Se ha mantenido constante? }\end{array}$ \\
\hline & & Operaciones & $\begin{array}{l}\text { ¿Tuvo retrasos al adquirir sus } \\
\text { suministros? } \\
\text { En caso de que la respuesta sea } \\
\text { afirmativa, ¿Tuvo que buscar } \\
\text { sustitutos? ¿Dónde los obtuvo? } \\
\text { ¿Se elevó el precio? }\end{array}$ \\
\hline & & Digitalización & $\begin{array}{l}\text { ¿Su empresa ya contaba con algún } \\
\text { servicio de digitalización? } \\
\mathrm{Si} \text {, No. } \\
\text { En caso afirmativo, ¿Cuál? } \\
\text { En caso negativo, ¿Platícanos qué } \\
\text { fue lo que hizo que no accediera a } \\
\text { la parte de la digitalización? }\end{array}$ \\
\hline & & Personal & $\begin{array}{l}\text { ¿Se vio en la necesidad de } \\
\text { rescindir contratos laborales a } \\
\text { personas dentro de su empresa? } \\
\text { ¿Mantuvo la nómina? } \\
\text { ¿Se disminuyeron los salarios? } \\
\text { ¿Se contrató a más personal? } \\
\text { ¿Redujo horas laborales? }\end{array}$ \\
\hline
\end{tabular}




\begin{tabular}{|c|c|c|c|}
\hline Identidad emprendedora & $\begin{array}{l}\text { La identidad emprendedora } \\
\text { se define como: Proceso } \\
\text { multidimensional de } \\
\text { construcción personal e } \\
\text { identificación axiológica, } \\
\text { dinámico e inacabado que se } \\
\text { desarrolla al vivir } \\
\text { situaciones ren } \\
\text { determinadas características } \\
\text { y condiciones en contextos } \\
\text { de interacción social, para } \\
\text { integrar las expectativas } \\
\text { personales y profesionales } \\
\text { con las experiencias vividas } \\
\text { para favorecer la } \\
\text { comprensión del sentido y } \\
\text { alcance de la competencia } \\
\text { emprendedora. } \\
\text { Glade (1969), Roberts y } \\
\text { Wood (2005), Cantón, Luit } \\
\text { y Ramirez (2008), Vázquez } \\
\text { y Dávila (2008), Bernal } \\
\text { (2014) y Navarrete (2015), } \\
\text { Martínez, N., y Rubio, B. } \\
\text { (2020) }\end{array}$ & Multidimensional & $\begin{array}{l}\text { ¿Reconoces tu perfil } \\
\text { emprendedor? } \\
\text { ¿Cuál es? } \\
\text { ¿Qué características tiene? } \\
\text { ¿Cómo se diferencia de otros tipos } \\
\text { de emprendedores? } \\
\text { ¿Cómo maneja el stress? } \\
\text { Platícanos, ¿Qué actitud toma ante } \\
\text { las crisis? }\end{array}$ \\
\hline $\begin{array}{l}\text { Necesidades formativas } \\
\text { para el emprendimiento }\end{array}$ & $\begin{array}{l}\text { Las necesidades de } \\
\text { formación para el } \\
\text { emprendimiento } \\
\text { reconocen a partir de las } \\
\text { competencias que se } \\
\text { requieren desarrollo en la } \\
\text { persona mediante procesos } \\
\text { que favorecen el encuentro, } \\
\text { la construcción y vivencia } \\
\text { de situaciones que } \\
\text { movilizan recursos para el } \\
\text { desarrollo de competencias } \\
\text { emprendedoras para } \\
\text { enfrentar las condiciones del } \\
\text { contexto de la pandemia por } \\
\text { Covid-19 } \\
\text { Contreras y Pérez (2013), de } \\
\text { Camilloni, (s/f), Ramirez } \\
\text { (2015), Osorio y Pereira } \\
\text { (2011) }\end{array}$ & Fines & $\begin{array}{l}\text { ¿Tuvo acceso a algún tipo de } \\
\text { formación durante y post } \\
\text { pandemia? } \\
\text {-Financiero } \\
\text {-Impuestos } \\
\text {-Recursos Humanos } \\
\text {-Manejo de stress } \\
\text { Podría mencionar ¿Cuál fue la } \\
\text { experiencia educativa más } \\
\text { significativa o enriquecedora } \\
\text { durante tu formación } \\
\text { emprendedora? y ¿Por qué? } \\
\text { ¿Cuál cree que fue la intención de } \\
\text { realizar estas actividades como } \\
\text { parte de su formación } \\
\text { emprendedora? } \\
\text { ¿Qué fue lo más importante para } \\
\text { usted? ¿Cuál fue el propósito que tuvo } \\
\text { para dicha actividad? }\end{array}$ \\
\hline
\end{tabular}

Fuente: Elaboración propia (2021) 


\section{REFERENCIAS}

Aguilar, J. (2020). México: efectos del COVID-19 en el mercado del trabajo. Resonancias. Blog de investigaciones Sociales de la UNAM. https://www.iis.unam.mx/blog/mexico-efectos-delcovid-19-en-el-mercado-del-trabajo/

Bernal, A. (2014). Competencia emprendedora e identidad personal. Una investigación exploratoria con estudiantes de Educación Secundaria Obligatoria. Revista de Educación, 363, 384-411.

Contreras, J. y Pérez, N. (2013). Investigar la experiencia educativa. Segunda edición. Morata. Madrid.

Camilloni A. (2013). La inclusión de la educación experiencial en el currículo universitario. En Menéndez, G. y otros, Integración docencia y extensión. Otra forma de aprender y de enseñar pp. 11-21. Santa Fe: Ediciones UNL.

Croda, G., y Sánchez, G. ( 2019). Construcción y diseño de un instrumento sobre experiencias educativas y la identidad del emprendedor en la formación universitaria. Memoria electrónica del CNIE. http://www.comie.org.mx/congreso/memoriaelectronica/v15/doc/3784.pdf

De la Vega, I. y Lorenzo, R. (2020). El emprendimiento y las pymes en tiempos de coronavirus.Revista Forbes. https://www.forbes.com.mx/el-emprendimiento-y-las-pymes-entiempos-de-coronavirus/

Esquivel, G. (2020). Los impactos económicos de la pandemia en México. Journal of Economic Literature. file:///Users/anadominguez/Downloads/543-Texto\%20de1\%20art\%C3\%ADculo1272-1-10-20200813.pdf

Gamez, Macías L.F. (2019). El emprendimiento en 2019. El financiero. https://www.elfinanciero.com.mx/bajio/el-emprendimiento-en-2019/

Maraboto, Moreno M. ( 2020). El efecto COVID-19 en las pymes. Expansión. https://expansion.mx/opinion/2020/06/12/el-efecto-covid-19-en-las-pymes

Muñoz Palacios, G. (2017). Emprender: alternativa profesional en la crisis del siglo XXI. Universidad de Valladolid. http://uvadoc.uva.es/handle/10324/27439

Nicolás Martínez, C., \& Rubio Bañón, A. (2020). Entrepreneurship in times of crisis: An exploratory analysis of the COVID-19's effects. Small Business International Review, 4(2), 53-66. https://doi.org/10.26784/sbir.v4i2.279

Navarrete, Baéz F.E. (2020). El COVID-19 y el impacto en micro empresas en México. UNIVA la Universidad Católica. https://www.univa.mx/guadalajara/el-covid-19-y-el-impacto-en-microempresas-en-mexico/

Navarrete, Z (2008). Proceso de construcción identitaria del pedagogo universitario en México. En Cadernos de Pesquisa. Departamento de Investigaciones en Educación del Centro de Investigación y de Estudios Avanzados del Instituto Politécnico Nacional. México. 38 (134), pp. 503-533.

Navarrete, Z. (2007). Constitución de sujetos: la identidad profesional del pedagogo. En memorias del IX Congreso Nacional de Investigación Educativa. México, COMIE.

Navarrete, Z. (2007). Identidad profesional del pedagogo universitario. Revista de las Facultades de Pedagogía de la Universidad Veracruzana. Xalapa. Pampedia, 3,12-21. https://www.academia.edu/6709144/Identidad_profesional_del_pedagogo_universitario

Osorio, F. y Pereira, F. (2011). Hacia un modelo de educación para el emprendimiento: una mirada desde la teoría social cognitiva. En Cuad. Admon.Ser.Organ. 24 (4), 13-33. Bogotá. 
Ramírez, J. (2015). Notas acerca de la noción de experiencia educativa. En Educación y ciudad. 11, $119-136$

Rodríguez, A. (2020). Cómo emprender en tiempos de crisis ¿Se acabaron las "vacas gordas"? A pesar de los desafíos, las circunstancias difíciles también abren oportunidades en Latinoamérica. Revista Entrepreneur. https://www.entrepreneur.com/article/269185

Zurlinden, P. (2010) La identidad y el aprendizaje: una perspectiva social. Multdisciplin@, 6, 5-13. http://www.revistas.unam.mx/index.php/multidisciplina/article/view/27792/25727 